\title{
ANDES

www.scielo.cl

\section{Profesionalismo y auto cuidado de los residentes, ¿cómo debemos enfocar su formación?}

\author{
Professionalism and self-care in residents, how should we confront their training?
}

\author{
Carla Benaglio $^{\mathrm{a}}$, Andrés Maturana ${ }^{\mathrm{a}, \mathrm{b}}$, Luigina Mortaric $^{\mathrm{c}}$, Arnoldo Riquelme $^{\mathrm{d}}$ \\ ${ }^{a}$ Centro de Desarrollo Educacional, Facultad de Medicina, Clínica Alemana-Universidad del Desarrollo. Santiago, Chile

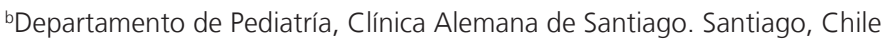 \\ 'Departamento de Educación, Universidad de Verona. Italia \\ ¿Departamento de Gastroenterología, Escuela de Medicina, Pontificia Universidad Católica de Chile. Santiago, Chile
}

Recibido el 2 de agosto de 2021; aceptado el 11 de agosto de 2021

¿Qué se sabe del tema que trata este estudio?

Cuando hablamos de profesionalismo en salud, el foco está puesto en el contrato social con nuestros pacientes y pares en el que esta implícita una lista de atributos deseables en el profesional de la salud. Sabemos que es una competencia que no se mantiene estable en el tiempo siendo muy sensible al bienestar del profesional.

\begin{abstract}
¿Qué aporta este estudio a lo ya conocido?
Exploramos la relación que emerge entre el profesionalismo y el auto cuidado para residentes en formación y como influyen el cansancio y agotamiento. El profesionalismo impacta en todas las otras competencias y se nutre de la autorreflexión. Se proponen una serie de recomendaciones que las instituciones formativas y sanitarias pueden considerar para cuidar el profesionalismo.
\end{abstract}

\section{Resumen}

El profesionalismo es una competencia bien definida en la educación de los residentes, sin embargo, es un constructo complejo, sensible a variables sociales y culturales. Puede definirse como el nivel de destreza, buen juicio y comportamiento adecuado esperable de personas entrenadas para realizar bien su trabajo. Es una competencia que no se mantiene estable en el tiempo y decae cuando el profesional está sometido a altos niveles de estrés y se asocia a calidad de cuidado, educación, ética, moral, filosofía y humanismo. Es una competencia esencial en el profesional por lo cual debemos replantear los currículos para incluir formas de enseñar y evaluar el profesionalismo. Es fundamental pensar en programas que logren equilibrar la carga de trabajo con el bienestar de los futuros profesionales. Debemos generar un clima adecuado de aprendizaje donde el profesional en entrenamiento sea un protagonista activo, y el auto cuidado sea visibilizado como una competencia esencial para mantener el equilibrio entre la vida personal y profesional. Este articulo plantea una revisión y reflexión de este tema que cobra cada vez mas importancia en la formación de postgrado de futuros especialistas.
Palabras clave:

Profesionalismo;

Educación

de Residente;

Autocuidado;

COVID-19;

Educación Médica

Correspondencia:

Carla Benaglio

cbenaglio@udd.cl 


\begin{abstract}
Professionalism is a well-defined competence in the education of residents. However, it is a complex construct, sensitive to social and cultural variables. It can be defined as the necessary skill, good judgment and appropriate behavior expected of people trained to do their jobs well. It is a competence that does not remain stable over time and declines when the professional is subjected to high levels of stress, associated with quality of care, education, ethics, moral, philosophy and humanism. It is an essential competence for the professional and therefore we must rethink the curricula to include ways to teach and evaluate professionalism. It is essential to design programs that balance the workload with the well-being of future professionals. We must generate an adequate learning environment where the trainee is an active protagonist, and self-care is made visible as an essential competence to maintain the balance between personal and professional life. This article presents a review and reflects on this topic which is becoming increasingly important in the postgraduate training of future specialists.
\end{abstract}

\section{Keywords:}

Professionalism;

Resident Education;

Self-Care;

COVID-19;

Medical Education

\section{Introducción}

El profesionalismo es una de las seis competencias principales en el profesional medico definidas por el Accreditation Council for Graduate Medical Education $(\mathrm{ACGME})^{1}$ para los estudiantes residentes y fellows, y por el American Academy of Pediatrics (AAP) y por American Board of Pediatrics (ABP $)^{2}$. Sin embargo, definirla no ha sido fácil, y sigue siendo una de las más complejas y controversiales, y frecuentemente la opinión pública denuncia comportamientos de profesionales de la salud que atentan contra esta competencia ${ }^{3}$.

¿Por qué hablar de profesionalismo cuando existe una vasta literatura sobre el tema? Porque el comportamiento profesional, o sea el mantener una conducta ética y excelente sigue siendo la competencia que tiene más impacto en la calidad del cuidado clínico, en la educación e investigación. Un ambiente donde no haya respeto en las relaciones, buena comunicación, y actitud de auto crítica entre los integrantes del equipo, no permite una buena practica clínica ${ }^{4}$.

La época que estamos viviendo de alto estrés determinado por la pandemia por COVID-19 obliga a una reflexión sobre el tema.

Un pediatra en urgencia después de 8 horas consecutivas de trabajo en urgencia calcula una dosis para una paciente pediátrica con una infección que a su parecer amerita tratamiento antibiótico. Por error calcula la dosis diaria total por $\mathrm{kg}$ de peso y la indica así para cada dosis olvidándola dividirla por el número de dosis al día. Le pasa la receta al residente en formación que está rotando por la urgencia y le solicita que se la entregue al paciente que acaban de examinar juntos. El residente ve la dosis y se da cuenta de que es el doble de lo que corresponde. Por temor a la reacción del médico decide conseguirse otra receta con otro médico de turno con la dosis correcta y no enfrentar la situación. Al finalizar el turno se cuestiona si hizo lo correcto.
En 1979 aparece en PubMed el termino MeSH Professional Competence (competencia profesional) definido como la capacidad de realizar las labores de la propia profesión en forma general o realizar una tarea profesional particular con una destreza aceptable. Solo recientemente (2016) aparece el termino profesionalismo definido como el nivel de destreza, buen juicio y comportamiento adecuado esperable de personas entrenadas para realizar bien su trabajo. Este se asocia a calidad de cuidado, educación, ética, moral, filosofía y humanismo, entre otros, y está fuertemente asociado a calidad y altos estándares del cuidado.

En 1999 la ACGME define las competencias principales (core competences) apareciendo por primera vez el concepto de profesionalismo en forma más explicita, centrado en la relación con el paciente 5 . La AAP y ABP hacen referencia a la definición de Stern del 2006 "Profesionalismo se demuestra a través de capacidad clínica, habilidades comunicacionales, y comprensión ética sobre lo que se construye la aspiración y sabia aplicación de los principios del profesionalismo: excelencia, humanismo, responsabilidad y altruismo". Lo anterior lleva las instituciones a focalizar la atención prioritariamente en el componente relacional, en la excelencia y en el rol social del médico ${ }^{6}$.

Las publicaciones sobre profesionalismo se han focalizado en medir empatía y en el evidenciar las dificultades en la relación con el paciente tanto en médicos formados como en formación ${ }^{7}$. Si bien el profesionalismo es un constructo muy amplio, el foco ha sido sobretodo la relación médico paciente. Desde los orígenes de la profesión médica se ha centrado en el otro, en particular en la comunicación, empatía, y cuidado centrado en el paciente ${ }^{8}$, el conocimiento científico y en valores que deberían guiar la acción del médico como altruismo, humanismo, vocación de servicio y compasión ${ }^{3}$.

La literatura revela claramente una erosión de este 
humanísimo inicial y un fuerte énfasis en la competencia técnica, que se refleja en currículos centrados en formar profesionales muy competentes desde el punto de vista técnico, sacrificando la enseñanza y evaluación del comportamiento9.

Existen otros aspectos que influyen en este proceso. El modelaje, como proceso informal y tradicional de socialización del rol profesional, se hace hoy insuficiente para garantizar la transmisión de los valores profesionales ${ }^{10}$ por una parte por lo complejo de la práctica médica y por otra por la heterogeneidad en los estudiantes de medicina ${ }^{11}$. En los últimos quince años se evidencia un progresivo aumento de problemas de salud mental en profesionales de la salud ${ }^{12-14}$, en particular entre los médicos y los residentes hay un elevado nivel de burnout, estrés y enfermedades mentales ${ }^{15}$. Se describen casos de suicidio en médicos y residentes en mayor frecuencia que en la población general ${ }^{16}$. La literatura reporta una prevalencia de burnout entre los estudiantes residentes de pediatria de 55 a $76 \%{ }^{17}$.

Durante la pandemia por COVID-19 esta prevalencia ha aumentado entre los profesionales de la salud por su alta exposición a altos niveles de estrés ${ }^{18}$.

En el ámbito académico y docente se evidencia un malestar general derivado de un ambiente altamente complejo con currículos muy exigentes que no siempre se han adaptado a esta nueva realidad y que persisten vinculados a un paradigma científico que ofrece pocos espacios para una formación humanista centrada en la persona. La organización de los procesos hace que los residentes vivencien su experiencia de aprendizaje con mucha ansiedad y preocupación. Se suma a esto un ambiente muy competitivo, sobretodo en etapas de transición (por ejemplo, de médico a especialista), y en particular en los programas de formación de especialidades de cirugía ${ }^{19-21}$. En este escenario hay experiencias reportadas como la inserción de cursos de atención plena y bienestar para reducir la ansiedad y mejorar la salud del residente ${ }^{22,23}$. Aun el impacto de estas intervenciones no ha sido adecuadamente evaluado sobretodo a largo plazo.

En época de pandemia por COVID-19, las publicaciones sobre el malestar de los equipos de salud y de los residentes han aumentado exponencialmente, caracterizándose como un periodo traumático ${ }^{18,24}$.

El objetivo de este manuscrito es proponer un marco referencial para reflexionar sobre la importancia del auto cuidado y bienestar mostrando su vinculación con el profesionalismo en los futuros especialistas.

\section{El profesionalismo: un constructo social}

Hablar de profesionalismo implica abrir un gran debate determinado por la ambigüedad y los distintos significados que se atribuyen a la palabra profesión y profesional para un constructo que tiene un gran impacto en la sociedad por las expectativas relativas a los comportamientos y actitudes de los profesionales. Representa una de las competencias más difíciles da definir, enseñar y evaluar por la complejidad de su naturaleza y su especificidad relativa al contexto ${ }^{25}$. En los últimos treinta años, ha tenido tantas variaciones e influencias que se ha convertido en un nuevo y muy complejo sistema social ${ }^{25}$. Si se considera un concepto dinámico que se sitúa en un contexto especifico, se puede comprender mejor su complejidad interna.

Es interesante destacar la publicación de Hafferty \& Castellani, donde se propone un análisis de la evolución histórica del concepto identificando distintos constructos. En particular el profesionalismo puede quedar en un ideal nostálgico hacia los valores y la relación médico-paciente, mientras hoy hay la necesidad de un nuevo equilibrio, en particular las nuevas generaciones reclaman la importancia de su bienestar y del tiempo libre, sobretodo con el ingreso cada día mayor de mujeres en las escuelas de especialidad. En cualquier conversación o definición de profesionalismo se debería considerar que el contexto, el lugar geográfico y la cultura local tienen un rol clave. No existe un único concepto de profesionalismo médico universalmente reconocido ${ }^{26,27}$. La definición propuesta por el American Board of Internal Medicine (ABIM) incluye seis dominios: altruismo, responsabilidad, excelencia, deber, honor, integridad y respeto hacia los otros. Sin embargo, esta propuesta no sintoniza con los valores de otras culturas no occidentales, donde armonizar la vida profesional con la vida personal es un valor importante ${ }^{11}$.

Otra propuesta, presenta cuatro niveles de compromiso profesional. Los compromisos con uno mismo, con las tareas propias de la profesión u oficio, con los demás, y con los propios valores o creencias ${ }^{11}$. Este modelo interpreta la responsabilidad, el compromiso con uno mismo y la automotivación desde una perspectiva de la fidelidad, donde en el contexto árabe de donde nace, la fe representa la base de los valores sociales.

La gran variabilidad cultural y la especificidad de cada contexto deberían estimular las escuelas y los equipos a desarrollar un modelo auténtico y con sentido para que pueda representar cada ambiente. Debería ser un modelo socializado y construido con los residentes para que tenga sentido y pueda ser enseñado y evaluado con una real integración de los valores y principios inspiradores. Además, considerando que el profesionalismo está relacionado con la cultura y el ambiente de trabajo, para que pueda accionar a nivel del currículo oculto, requiere definiciones y proyectos coherentes a nivel institucional ${ }^{28}$. Adicionalmente, requiere que los profesionales de la salud como grupo 
definan estándares comunes para esta competencia que gobierna el trabajo medico ${ }^{29}$.

\section{Profesionalismo y auto cuidado: un imperativo ético para el profesional y su paciente}

El profesionalismo es una competencia multidimensional, sensible a características como autoconocimiento, presencia, hábitos, atención y curiosidad critica. Es una competencia que se desarrolla en el tiempo, que no es permanente, se modifica a lo largo de la vida profesional y necesita de una constante preocupación y atención'. La ausencia de autocuidado puede llevar al agotamiento o "burnout" que demostradamente erosiona el nivel y la estabilidad del profesionalismo ${ }^{30,31}$. El comportamiento profesional es particularmente vulnerable durante los periodos de extremo estrés, ansiedad y "burnout"17.

El bienestar del profesional médico tiene un impacto en el cuidado del paciente. Está demostrado que un clínico con menor capacidad de autocuidado parece tener menor capacidad de cuidar a otra persona y comete más errores ${ }^{30}$. Por otro lado, un residente en formación que incorpora actividades de auto cuidado en su routina diaria es más resiliente y tendria un menor riesgo de colapsar durante su vida académica ${ }^{32}$. El residente está más expuesto a situaciones de alto estrés que son el resultado de una combinación de factores que incluyen la falta de horas de descanso, la presión de su ambiente de trabajo, y su vulnerabilidad (por una menor experiencia y conocimiento) relativos al proceso de formación ${ }^{33}$. Cuando la capacidad para soportar el estrés se reduce, se compromete el bienestar y las personas pueden manifestar comportamientos no profesionales que no aparecerían en circumstancias habituales. El poder conversar de estos temas es prioritario para profesionales en formación, en particular para el desarrollo de su identidad profesional y para adquirir estrategias que sean útiles para toda su carrera profesional ${ }^{17}$.

La construcción de la identidad profesional es un proceso complejo que se desarrolla en los hospitales durante el trabajo diario, sobretodo la interacción y trabajo en equipo, donde las exigencias que expresan los residentes en relación con el tiempo protegido para el aprendizaje se interpretan como debilidad y como una evasión de sus responsabilidades ${ }^{34,35}$. Además, los residentes perciben que solicitar respeto por sus horarios o por su tiempo libre no es interpretado por sus tutores como autocuidado ${ }^{36}$. Los residentes manifiestan dificultad para pedir ayuda, en particular por problemas de salud mental que son fuertemente estigmatiza$\operatorname{dos}^{37}$. El ambiente clínico es altamente complejo, y en algunos casos los residentes perciben discriminación, crítica constante o abuso de su tiempo ${ }^{38}$. Lo que revela la disonancia en el marco de referencia que cada grupo tiene en relación con el profesionalismo: los conflictos que se producen son entre un modelo de profesionalismo que se centra en el deber ser y el contrato social, mas propio en docentes y tutores, versus un modelo que integra el auto cuidado con un mayor equilibrio entre la vida profesional y la vida personal ${ }^{39}$.

Durante el periodo de pandemia por COVID-19, donde los equipos de salud se han visto expuestos a un significativo mayor agotamiento emocional, en el caso de los residentes, ha aumentado el riesgo de comprometer su salud física y mental ${ }^{40}$.

Si retornamos a la situación descrita en la viñeta: El temor y la ausencia de un espacio de confianza impiden al residente conversar libremente con el médico que lo supervisa y eso lo lleva a encubrir el error. El crear un espacio seguro para conversar sobre temas dificiles como este, protege a los pacientes y también al mismo profesional, siempre que exista una instancia de reflexión constructiva, y no castigadora.

La falta de comunicación, un ambiente complejo, el "burnout" y el malestar disminuyen el profesionalismo y potencialmente pueden tener un fuerte impacto en el paciente aumentando la posibilidad de errores y mala praxis ${ }^{41}$. Además, influyen en los residentes en la relación con su satisfacción por el trabajo y especialidad futura ${ }^{20,42}$.

\section{Estrategias para enseñar profesionalismo y auto cuidado durante la pandemia de COVID-19}

Si bien nadie estaba preparado para enfrentar el estrés asociado a esta pandemia, algunas escuelas de postgrado implementaron rápidamente programas para mejorar el bienestar de sus estudiantes ${ }^{43}$. En base a la retroalimentación de los residentes, se implementaron cambios en el currículo y en la metodología docente. La pandemia ha acelerado y en ocasiones forzado cambios en un contexto con niveles de estrés inusualmente altos y transversales a todos. Los mismos residentes, sobretodo de los programas con menos exigencias en la primera línea y por ende con "más tiempo", han colaborado al introducir cambios importantes en la didáctica a distancia, y ofreciendo mayor supervisión y apoyo a los residentes que atienden pacientes COVID-19 $9^{44-46}$.

Algunos centros han implementado cambios orientados a hacer mejor uso del tiempo, sustituyendo las clases con video conferencias, introduciendo reuniones semanales de mentore ${ }^{40}$, y organizando más espacios de docencia asincrónica ${ }^{47}$. Esto ha permitido reorganizar la didáctica dejando más espacio al trabajo 
clínico y al descanso, planificando cursos más coherentes con el tiempo disponible y optimizando recursos y espacios educativos.

En algunas especialidades la reducción drástica del trabajo clínico de los residentes por la pandemia ha creado espacios de tiempo protegido para que puedan escribir y publicar sus tesis ${ }^{48}$.

Durante la pandemia ha sucedido otro fenómeno importante para el profesionalismo y el auto cuidado: una explosión de narrativas, fotos, historias y experiencias de los profesionales de la salud publicadas en diversos medios relacionadas con una vivencia de fuerte estrés. Los clínicos han encontrado en la narrativa un espacio para recuperar un poco de bienestar. La experiencia clínica con el COVID-19 habla de shock y de trauma $^{18}$, sin embargo, emerge también la fuerza de los equipos, el valor de la experiencia compartida y de la escritura $^{49}$. Por lo tanto, el enriquecer los programas de especialidad y subespecialidad con espacios de reflexión usando la literatura y la narrativa impacta positivamente sobre el bienestar y el auto cuidado ${ }^{50,51}$. La reflexión sobre las historias reales o ficticias, de enfermedad o de otros aspectos relacionados con la experiencia del ser humano, ofrece un antídoto a la insatisfacción y conecta al residente con su motivación original para cuidar a otro $^{52}$. La medicina narrativa, en particular el método implementado por la Rita Charon, lectura atenta (close reading) y la escritura reflexiva (reflective writing) ofrecen un espacio para reflexionar sobre las experiencias más difíciles y traumáticas de la relación con el otro y la fatiga que esto puede generar ${ }^{53,54}$. Introducir espacios de reflexión impacta sobre el bienestar del médico en formación y su relación con el paciente ${ }^{55-57}$.

Cómo replantear la formación de los médicos en relación con el profesionalismo y al auto cuidado: algunas recomendaciones

1. El profesionalismo es un constructo complejo, y debemos replantear la forma para enseñar y evaluar el profesionalismo ${ }^{31}$.

2. Los estudiantes deberían ser protagonistas activos de su aprendizaje del profesionalismo incorporándose en la docencia y colaborando activamente ${ }^{40}$.

3. Los docentes deberían generar instancias de conversación y discusión de temas como el bienestar y auto cuidado. Deberían ser activos en modelar conductas profesionales sanas ${ }^{58}$.

4. El auto cuidado debería ser considerado una competencia explicita y bien definida da enseñar con cursos en los programas de postgrado ${ }^{43}$.

5. Las instituciones deberían promover una cultura de confianza, respeto, trabajo en equipo, buena comunicación, y enfrentamiento de errores en una manera no amenazante. Deben promover el respeto mutuo y la confianza entre profesionales previniendo el agotamiento ${ }^{39}$.
6. El profesionalismo es una competencia que impacta en todas las demás. Este decae en el tiempo y está relacionada con menor riesgo de cometer errores. Debemos garantizar la mantención de esta competencia con algunos indicadores mínimos que pueden retroalimentar el residente y el equipo de salud $^{31}$.

7. Una comunidad que se cuida no se etiqueta: hablar con los residentes y los equipos médicos de los problemas de salud mental; abrir las conversaciones sobre las temáticas más difíciles y crear programas de apoyo que mantengan la confidencialidad protege a los futuros especialistas ${ }^{36}$.

8. Introducir las humanidades como espacio para enfrentar las situaciones profesionales más complejas y como método para el auto cuidado ${ }^{51}$.

9. Es necesaria formación continua en relación con el profesionalismo, al cuidado de los equipos y al auto cuidado de cada integrante; la formación ofrece espacios de reflexión y discusión. Y eso transforma las experiencias en aprendizaje, y aumenta la competencia. Los Centros de Educación Médica deberían considerar el profesionalismo y el bienestar entre los cursos que ofrecen a sus acadé$\operatorname{micos}^{55}$.

10. Repensar a los programas de postgrado, colaborar, flexibilizar, integrar los aprendizajes que derivan de la época de la pandemia. Innovar para proteger el tiempo del residente ${ }^{46}$.

\section{Conclusiones}

Para mantener el profesionalismo de los futuros especialistas es fundamental pensar en programas que logren equilibrar las exigencias con el bienestar. Fomentar una educación donde el residente sea el protagonista y sea acompañado de tutores formados y focalizados en su aprendizaje. Deben ser modelos para el residente en formación de como equilibrar la vida personal con la responsabilidad profesional sin perder de vista sus valores y principios inspiradores. Una educación que fomente la responsabilidad del futuro especialista en su auto aprendizaje, que lo considere un protagonista activo, donde el auto cuidado sea visibilizado como una competencia esencial para mantener el equilibrio entre la vida personal y profesional. El equipo donde está inserto el residente tiene un rol clave para validar las emociones que derivan de cuidados que pueden ser muy complejos y que pueden generar agotamiento emocional. Es importante que el profesional en formación encuentre un real espacio de conversación y escucha donde exista tiempo protegido para discutir situaciones como la planteada en el caso relatado. Un clima inadecuado de aprendi- 
zaje que induzca a no enfrentar, o peor aun, ocultar situaciones relevantes como el error (como en la viñeta presentada), puede perjudicar tanto a los profesionales involucrados como a sus pacientes. Debemos proyectarnos hacia una fase post pandemia, con una comunidad profesional resiliente y empática con sus pares, atesorando los aprendizajes del COVID-19 en educación.

\section{Conflicto de intereses}

Los autores declaran no tener conflicto de intereses.

\section{Referencias}

1. Birden H, Nass D. Teaching Professionalism in medical education: A best evidence in Medical Education (BEME) systematic review. BEME Guide No. 25. Med Teach. 2013;35(7):e1252ele66. (https://www.tandfonline.com/ doi/full/10.3109/0142159X.2013.789132. Accedido 16.07.2021).

2. Kirk LM. Professionalism in Medicine: Definitions and Considerations for Teaching. Bayl Univ Med Cent Proc. 2007;20(1):13-6. (https://www. tandfonline.com/doi/abs/10.1080/089982 80.2007.11928225. Accedido 20.07.2020)

3. Blanco M, Maderer A, Price L, Epstein S, Summergrad P. Efficiency is not enough; you have to prove that you care: Role modelling of compassionate care in an innovative resident-as-teacher initiative. Educ Health. 2013;26(1):60. (https:// www.educationforhealth.net/article.asp. Accedido 29.04.2020)

4. Waddell JP, Warnock GL. Reflecting on principles of professionalism. Can J Surg. 2008;51(2): 84-85. (https://www.ncbi. nlm.nih.gov/pmc/articles/PMC2386327/. Accedido 24.06.2021)

5. Frank JR, Snell L, Sherbino J, Royal College of Physicians and Surgeons of Canada. CanMEDS 2015: physician competency framework. 2015. (https:// www.royalcollege.ca/rcsite/documents/ canmeds/canmeds-full-framework-e.pdf. Accedido 19.04.2020)

6. Stern DT, Papadakis M. The Developing Physician - Becoming a Professional. N Engl J Med. 2006;355(17):1794-9. (https:// www.bumc.bu.edu/facdev-medicine/ files/2010/06/Stern-NEJM-2006.pdf. Accedido 10.08.2020).

7. Dewa CS, Loong D, Bonato S, Trojanowski L, Rea M. The relationship between resident burnout and safetyrelated and acceptability-related quality of healthcare: a systematic literature review. BMC Med Educ. 2017;17(1):195. (https:// bmcmededuc.biomedcentral.com/track/ pdf/10.1186/s12909-017-1040-y.pdf. Accedido 29.04.2020)

8. Busireddy KR, Miller JA, Ellison K, Ren V, Qayyum R, Panda M. Efficacy of Interventions to Reduce Resident Physician Burnout: A Systematic Review.
J Grad Med Educ. 2017;9(3):294-301. (https://www.ncbi.nlm.nih.gov/pmc/ articles/PMC5476377/. Accedido 29.04.2020)

9. Charon R. Commentary: Our Heads Touch. Acad Med. 2012;87(9):1154-6. https://www.ncbi.nlm.nih.gov/pmc/ articles/PMC4021013/. Accedido 20.07.2020)

10. Epstein RM. Defining and Assessing Professional Competence. JAMA. 2002 ;287(2):226. (https://www.researchgate. net/publication/298348201_Defining and_Assessing_Professional_Competence. Accedido 10.08.2020)

11. Fernanda B, Cabrera D, Sadosty A. et al. Compassion Fatigue is Similar in Emergency Medicine Residents Compared to other Medical and Surgical Specialties. West J Emerg Med. 2014;15(6):62935. (https://www.ncbi.nlm.nih.gov/ pmc/articles/PMC4162717/. Accedido 09.08.2020)

12. Al-Eraky MM. Twelve Tips for teaching medical professionalism at all levels of medical education. Med Teach. 2015;37(11):1018-25. (https://www. tandfonline.com/doi/abs/10.3109/01421 59X.2015.1020288? journalCode=imte20. Accedido 10.01.2021)

13. Aherne D, Farrant K, Hickey L, Hickey E, McGrath L, McGrath D. Mindfulness based stress reduction for medical students: optimising student satisfaction and engagement. BMC Med Educ. 2016;16(1):209. (https://bmcmededuc. biomedcentral.com/articles/10.1186/ s12909-016-0728-8. Accedido 14.09.2020)

14. Grendar J, Beran T, Oddone-Paolucci E. Experiences of pressure to conform in postgraduate medical education. BMC Med Educ. 2018;18(1):4. (https:// bmcmededuc.biomedcentral.com/ articles/10.1186/s12909-017-1108-8. Accedido 14.09.2020)

15. Beckman TJ, Reed DA, Shanafelt TD, West CP. Resident Physician Well-Being and Assessments of Their Knowledge and Clinical Performance. J Gen Intern Med. 2012;27(3):325-30. (https://www.ncbi. nlm.nih.gov/pmc/articles/PMC3286555/. Accedido 29.04.2020)

16. Minford EJ, Manning CL. Current status and attitudes to self-care training in UK medical schools. J Compassionate
Health Care. 2017;4(1):3. (https:// jcompassionatehc.biomedcentral.com/ articles/10.1186/s40639-017-0032-4. Accedido 14.09.2020)

17. McLuckie A, Matheson KM, Landers AL. et al. The Relationship Between Psychological Distress and Perception of Emotional Support in Medical Students and Residents and Implications for Educational Institutions. Acad Psychiatry. 2018;42(1):41-7. (https:// link.springer.com/article/10.1007 \%2Fs40596-017-0800-7. Accedido 14.09.2020)

18. Livingston EH, Ginsburg S, Levinson $\mathrm{W}$. Introducing JAMA Professionalism. JAMA. 2016;316(7):720. (https:// jamanetwork.com/journals/jama/ fullarticle/2544618. Accedido 13.01.2021)

19. Bennett $P$, Noble $S$, Johnston $S$, Jones D, Hunter R. COVID-19 confessions: a qualitative exploration of healthcare workers experiences of working with COVID-19. BMJ Open. 2020;10(12):e043949. (https://bmjopen. bmj.com/content/10/12/e043949. Accedido 03.01.2021)

20. Blackmore C, Austin J, Lopushinsky SR, Donnon T. Effects of Postgraduate Medical Education "Boot Camps" on Clinical Skills, Knowledge, and Confidence: A Meta-Analysis. J Grad Med Educ. 2014;6(4):643-52. (https:// www.ncbi.nlm.nih.gov/pmc/articles/ PMC4477555/. Accedido 14.09.2020)

21. Feddock CA, Hoellein AR, Wilson JF, Caudill TS, Griffith CH. Do pressure and fatigue influence resident job performance? Med Teach. 2007;29(5):495-7. (https://www. tandfonline.com/doi/abs/10.1080/0142 1590701506874 ? journalCode $=$ imte20. Accedido 03.05.2020)

22. Pulcrano M, Evans SRT, Sosin M. Quality of Life and Burnout Rates Across Surgical Specialties: A Systematic Review. JAMA Surg. 2016;151(10):970. (https:// jamanetwork.com/journals/jamasurgery/ article-abstract/2533102. Accedido 03.05.2020)

23. Daya Z, Hearn JH. Mindfulness interventions in medical education: A systematic review of their impact on medical student stress, depression, fatigue and burnout. Med Teach. 2018;40(2):146- 
53. (https://www.tandfonline.com/doi/abs /10.1080/0142159X.2017.1394999?journal Code $=$ imte20. Accedido 14.09.2020)

24. Scheepers RA, Emke H, Epstein RM, Lombarts KMJMH. The impact of mindfulness-based interventions on doctors' well-being and performance: A systematic review. Med Educ. 2020;54(2):138-49. (https://www.ncbi. nlm.nih.gov/pmc/articles/PMC7003865/. Accedido 14.09.2020)

25. Treluyer L, Tourneux P. Burnout among paediatric residents during the COVID-19 outbreak in France. Eur J Pediatr. 2021;180(2):627-33. (https://link.springer. com/article/10.1007\%2Fs00431-02003907-x. Accedido 14.06.2021)

26. Castellani B, Hafferty FW. The Complexities of Medical Professionalism. In: Wear D, Aultman JM, editors. Professionalism in Medicine [Internet]. Boston: Kluwer Academic Publishers; 2006.

27. Arnold L, Stern DT. What Is Medical Professionalism?: p. 24. In: Stern DT editor. Measuring Medical Professionalism. New York: Oxford University Press; 2005.

28. Hilton SR, Slotnick HB. Protoprofessionalism: how professionalisation occurs across the continuum of medical education. Med Educ. 2005;39(1):5865. (https://onlinelibrary.wiley.com/ doi/10.1111/j.1365-2929.2004.02033.x. Accedido 10.01.2021)

29. Berger AS, Niedra E, Brooks SG, Ahmed WS, Ginsburg S. Teaching Professionalism in Postgraduate Medical Education: A Systematic Review. Acad Med. 2020;95(6):938-46. (https:// journals.lww.com/academicmedicine/ Fulltext/2020/06000/Teaching_ Professionalism_in_Postgraduate_ Medical.43. Accedido 14.09.2020)

30. Wynia MK, Papadakis MA, Sullivan WM, Hafferty FW. More Than a List of Values and Desired Behaviors: A Foundational Understanding of Medical Professionalism. Acad Med. 2014;89(5):712-4. (https:// journals.lww.com/academicmedicine/ Fulltext/2014/05000/More_Than_a_List_ of_Values_and_Desired_Behaviors_.13. aspx. Accedido 14.07.2021)

31. Conran RM, Powell SZ-E, Domen RE. et al. Development of Professionalism in Graduate Medical Education: A CaseBased Educational Approach From the College of American Pathologists' Graduate Medical Education Committee. Acad Pathol. 2018;5:237428951877349. (https://www.ncbi.nlm.nih.gov/pmc/ articles/PMC6039899/. Accedido 05.08.2020)

32. Ludwig S. Domain of Competence: Professionalism. Acad Pediatr.
2014;14(2):S66-9. (https://www. academicpedsjnl.net/article/S18762859(13)00328-8/fulltext. Accedido 10.08.2020)

33. Ayala EE, Winseman JS, Johnsen RD, Mason HRC. U.S. medical students who engage in self-care report less stress and higher quality of life. BMC Med Educ. 2018;18(1):189. (https://bmcmededuc. biomedcentral.com/articles/10.1186/ s12909-018-1296-x. Accedido 14.09.2020)

34. Wei JM, Fernandez-Salvador C, Camacho M. Health in Residency: The Dilemma of Caring for Yourself. J Grad Med Educ. 2017;9(5):670. (https://www.ncbi.nlm. nih.gov/pmc/articles/PMC5646939/. Accedido 29.04.2020)

35. Phillips SP, Dalgarno N. Professionalism, professionalization, expertise and compassion: a qualitative study of medical residents. BMC Med Educ. 2017;17(1):21. (https://www.ncbi.nlm.nih.gov/pmc/ articles/PMC5259915/. Accedido 29.04.2020)

36. Jarvis-Selinger S, Pratt DD, Regehr G. Competency Is Not Enough: Integrating identity formation into the medical education discourse. Acad Med. 2012;87(9):1185-90. (https:// journals.lww.com/academicmedicine/ Fulltext/2012/09000/Competency_Is_ Not_Enough_Integrating_Identity.16.a. Accedido 28.07.2020)

37. Bitran $M$, Zúñiga $D$, Pedrals $N$, Echeverría G, Vergara C, Rigotti A, et al. Burnout en la formación de profesionales de la salud en Chile: Factores de protección y riesgo, y propuestas de abordaje desde la perspectiva de los educadores. Rev Med Chil. 2019;147(4):510-7. (https://www. scielo.cl/scielo.php?script=sci_arttext\&pi $\mathrm{d}=$ S0034-98872019000400510. Accedido 19.03.2021)

38. Kassam A, Cowan M, Topps M. Lessons Learned to Aid in Developing Fatigue Risk Management Plans for Resident Physicians. Teach Learn Med. 2019;31(2):136-45. (https://www. tandfonline.com/doi/abs/10.1080/10401 334.2018.1542307?journalCode $=\mathrm{h} t \operatorname{lm} 20$. Accedido 14.09.2020)

39. Bitran M, González M, Nitsche P, Zúñiga D, Riquelme A. Preocupación por el bienestar de residentes, un tema presente en la Conferencia Latinoamericana en Educación de Residentes (LACRE) 2017. Rev Med Chil. 2017;145(10):13305. (https://scielo.conicyt.cl/scielo. php?script=sci_arttext\&pid=S003498872017001001330\&lng=es\&nrm=iso. Accedido 03.08.2020)

40. Ripp JA, Privitera MR, West CP, Leiter R, Logio L, Shapiro J, et al. Well-Being in Graduate Medical Education: A Call for Action. Acad Med. 2017;92(7):9147. (https://journals.lww.com/
academicmedicine/Fulltext/2017/07000/ Well_Being_in_Graduate_Medical_ Education. Accedido 14.09.2020)

41. Lie JJ, Huynh C, Scott TM, Karimuddin AA. Optimizing Resident Wellness During a Pandemic: University of British Columbia's General Surgery Program's COVID-19 Experience. J Surg Educ. 2021;78(2):366-9. (https://www.ncbi. nlm.nih.gov/pmc/articles/PMC7368914/. Accedido 22.05.2021)

42. Brennan MD, Monson V. Professionalism: Good for Patients and Health Care Organizations. Mayo Clin Proc. 2014;89(5):644-652. (https:// www.mayoclinicproceedings.org/article/ S0025-6196(14)00064-0/pdf. Accedido 19.04.2020)

43. Davenport DL, Henderson WG, Hogan S, Mentzer RM, Zwischenberger JB. Surgery resident working conditions and job satisfaction. Surgery. 2008;144(2):332338.e5. (http://europepmc.org/article/ MED/18656643. Accedido 03.05.2020)

44. Roytman M, Shah S. Lessons learned during the COVID-19 pandemic: a single institution radiology chief resident experience. Clin Imaging. 2020;68:903. (https://www.clinicalimaging.org/ article/S0899-7071(20)30234-5/fulltext. Accedido 18.06.2021)

45. Hau H-M, Weitz J, Bork U. Impact of the COVID-19 Pandemic on Student and Resident Teaching and Training in Surgical Oncology. J Clin Med. 2020;9(11):3431. (https://www.ncbi.nlm. nih.gov/pmc/articles/PMC7692303/. Accedido 02.01.2021)

46. Rabinowitz DG, Sundheim KM. Trainee-Directed Educational Pursuits and Advocacy During the COVID-19 Pandemic. Pediatrics. 2020;146(3):e20201564. (https:// pediatrics.aappublications.org/ content/146/3/e20201564.long. Accedido 02.01.2021)

47. Ricciardi G, Biondi R, Tamagnini G. Go back to the basics: Cardiac surgery residents at the time of COVID-19. J Card Surg. 2020;35(7):1400-2. (https:// www.ncbi.nlm.nih.gov/pmc/articles/ PMC7300607/. Accedido 02.01.2021)

48. Chertoff JD, Zarzour JG, Morgan DE, Lewis PJ, Canon CL, Harvey JA. The Early Influence and Effects of the Coronavirus Disease 2019 (COVID-19) Pandemic on Resident Education and Adaptations. J Am Coll Radiol. 2020;17(10):13228. (https://www.ncbi.nlm.nih.gov/ pmc/articles/PMC7375309/. Accedido 02.01.2021)

49. Degraeve A, Lejeune S, Muilwijk T, Poelaert F, Piraprez M, Svistakov I, et al. When residents work less, they feel better: Lessons learned from an unprecedent context of lockdown. Prog En Urol. 
2020;30(16):1060-6. (https://www.ncbi. nlm.nih.gov/pmc/articles/PMC7833413/. Accedido 15.06.2021)

50. Mayo AT. Teamwork in a pandemic: insights from management research. BMJ Lead. 2020;4(2):53-6. (https://bmjleader. bmj.com/content/4/2/53. Accedido 08.01. 2021)

51. Dotters-Katz S, Chuang A, Weil A, Howell J. Developing a pilot curriculum to foster humanism among graduate medical trainees. J Educ Health Promot. 2018;7(1):2. (https://www.ncbi.nlm.nih. gov/pmc/articles/PMC5791433/. Accedido 09.08.2020)

52. Arntfield SL, Slesar K, Dickson J, Charon R. Narrative medicine as a means of training medical students toward residency competencies. Patient Educ Couns. 2013;91(3):280-6. (https:// www.ncbi.nlm.nih.gov/pmc/articles/ PMC3992707/. Accedido 20.07.2020)

53. Lijoi AF, Tovar AD. Narrative medicine: Re-engaging and re-energizing ourselves through story. Int J Psychiatry Med. 2020;55(5):321-30. (https://journals. sagepub.com/doi/10.1177/009121742 0951039?url_ver=Z39.88-2003\&rfr_ id=ori:rid:crossref.org\&rfr_dat $=$. Accedido 14.09.2020)

54. Charon R. Narrative Medicine: A Model for Empathy, Reflection, Profession, and Trust. JAMA. 2001;286(15):1897. (https://jamanetwork. com/journals/jama/fullarticle/194300. Accedido 20.07.2020)

55. Charon R, Hermann N, Devlin MJ. Close Reading and Creative Writing in Clinical Education: Teaching Attention, Representation, and Affiliation. Acad Med. 2016;91(3):345-50. (https:// www.ncbi.nlm.nih.gov/pmc/articles/ PMC4721945/. Accedido 20.07.2020)

56. Montgomery L, Loue S, Stange KC. Linking the Heart and the Head: Humanism and Professionalism in Medical Education and Practice. Fam Med. 2017;49(5):378-383. (https://www. stfm.org/FamilyMedicine/Vol49Issue5/ Montgomery378. Accedido 20.07.2020)

57. Winkel AF, Hermann N, Graham MJ, Ratan RB. No Time to Think: Making
Room for Reflection in Obstetrics and Gynecology Residency. J Grad Med Educ. 2010;2(4):610-5. (https://www.ncbi. nlm.nih.gov/pmv/articles/PMC3010949. https://www.ncbi.nlm.nih.gov/pmc/ articles/PMC3010949/https://www.ncbi. nlm.nih.gov/pmc/articles/PMC3010949/ Accedido 31.07.2020)

58. Winkel AF, Honart AW, Robinson A, Jones A-A, Squires A. Thriving in scrubs: a qualitative study of resident resilience. Reprod Health. 2018;15(1):53. (https://reproductive-health-journal. biomedcentral.com/articles/10.1186/ s12978-018-0489-4. Accedido 03.08.2020)

59. Branch WT, Frankel R, Gracey CF, Haidet PM, Weissmann PF, Cantey $\mathrm{P}$, et al. A Good Clinician and a Caring Person: Longitudinal Faculty Development and the Enhancement of the Human Dimensions of Care: Acad Med. 2009;84(1):117-25. (https:// journals.lww.com/academicmedicine/ Fulltext/2009/01000/A_Good_Clinician_ and_a_Caring_Person_LLongitudinal. Accedido 03.05.2020) 